\title{
Three-dimensional speckle-tracking echocardiography for the global and regional assessments of left ventricle myocardial deformation in breast cancer patients treated with anthracyclines
}

\author{
Madalena Coutinho Cruz ${ }^{1}\left[\right.$ ] - Luísa Moura Branco ${ }^{1}$. Guilherme Portugal ${ }^{1}$. Ana Galrinho ${ }^{1}$. Ana Teresa Timóteo ${ }^{1}$. \\ Pedro Rio ${ }^{1} \cdot$ Rita Ilhão Moreira ${ }^{1}$. Tiago Mendonça ${ }^{1} \cdot$ Ana Leal $^{1}$ - Fernanda Gameiro ${ }^{1}$ Sónia Duarte Oliveira ${ }^{2}$. \\ Ricardo Luz $^{2} \cdot$ Rui Cruz Ferreira' ${ }^{1}$
}

Received: 27 July 2019 / Accepted: 17 September 2019 / Published online: 26 September 2019

(c) Springer-Verlag GmbH Germany, part of Springer Nature 2019

\begin{abstract}
Background Assessment of 2D/3D left ventricular ejection fraction (LVEF) and 2D global longitudinal strain (GLS) is the gold standard for diagnosing cancer therapeutics-related cardiac dysfunction (CTRCD). Although 3D speckle-tracking echocardiography (STE) has several advantages, it is not used in this setting.

Methods 105 breast cancer patients who underwent serial echocardiographic assessment during anthracycline therapy were included. STE was used to estimate 2D GLS, 3D GLS, 3D global circumferential strain (GCS), 3D global radial strain (GRS), and 3D global area strain (GAS). CTRCD was defined as an absolute decrease in 2D/3D LVEF $>10 \%$ to a value $<54 \%$ or a relative decrease in $2 \mathrm{D}$ GLS $>15 \%$.

Results 24 patients developed CTRCD. There was a significant worsening of all 3D strain parameters during chemotherapy. 3D strain regional analysis showed impaired contractility in the anterior, inferior, and septal walls. Variations of 3D GRS and 3D GCS were associated with a higher incidence of CTRCD and the variation of 3D GRS was an independent predictor of CTRCD. Variations of 3D GCS and 3D GRS had a good discrimination for predicting CTRCD, with optimal cutoff values of $-34.2 \%$ for 3D GCS and $-34.4 \%$ for 3D GRS. These variations were observed 45 and 23 days before the diagnosis of CTRCD, respectively.

Conclusion Variations of 3D strain parameters were predictive of and preceded CTRCD, and thus have added value over currently recommended 2D/3D LVEF and 2D GLS. Routine application of this technique should be considered to offer targeted monitoring and timely initiation of cardioprotective treatment.
\end{abstract}

Electronic supplementary material The online version of this article (https://doi.org/10.1007/s00392-019-01556-1) contains supplementary material, which is available to authorized users.

Madalena Coutinho Cruz

madalena.cruz89@gmail.com

1 Cardiology Department, Hospital de Santa Marta, Centro Hospitalar Lisboa Central, EPE, Rua de Santa Marta, 50, 1169-024 Lisbon, Portugal

2 Oncology Department, Hospital de Santo António dos Capuchos, Centro Hospitalar de Lisboa Central, EPE, Alameda de Santo António dos Capuchos, 1169-050 Lisbon, Portugal 


\section{Graphic abstract}

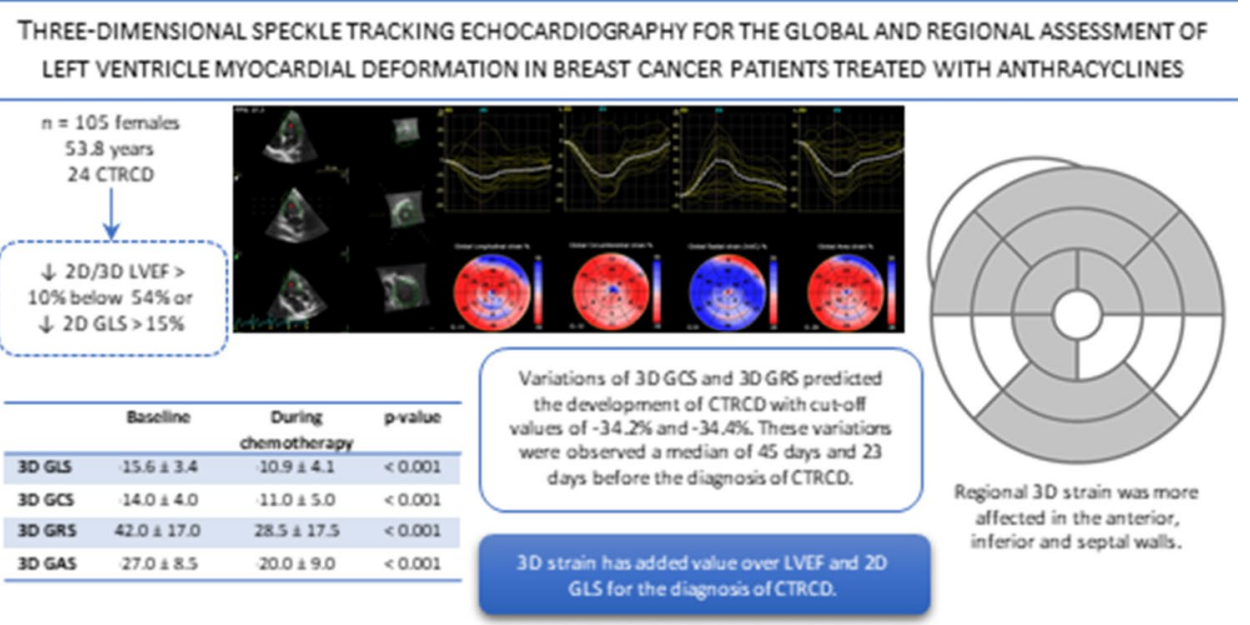

Keywords Anthracyclines $\cdot$ Cardiotoxicity $\cdot$ Speckle-tracking echocardiography $\cdot$ Strain $\cdot$ Three-dimensional

\section{Introduction}

Cancer therapeutics-related cardiac dysfunction (CTRCD) is a common complication of anthracycline treatment, affecting up to $26 \%$ of patients receiving standard cumulative doses [1], which can have a significant impact on patient outcomes [2]. Although there is universal agreement for the need to monitor cardiac function after exposure to anthracyclines, current surveillance approaches are often inadequate to detect myocardial disease; the issue of the best screening method remains unresolved.

Left ventricle ejection fraction (LVEF) has been the most widely used tool to diagnose cardiac dysfunction in this setting [3-5]. However, this method presents several shortcomings, such as the need for geometrical assumptions, apical foreshortening, load dependency, and measurement variability [6, 7]. Three-dimensional (3D) LVEF emerged as an alternative to overcome these problems, since it is less dependent on geometrical assumptions, has a greater reproducibility of sequential assessments and a better correlation with cardiac magnetic resonance [6-8]. A further limitation of LVEF is the fact that it fails to detect early changes in cardiac function, before an irreversible loss of myocardium has occurred $[5,9,10]$. Twodimensional (2D) speckle-tracking echocardiography (STE) with assessment of myocardial deformation is able to detect subclinical cardiac dysfunction [11]. These techniques are now recommended over 2D LVEF to screen for CTRCD [12].

3D STE has the ability to track out-of-plane motion of speckles, has a higher reproducibility of measurements, and allows for a simultaneous evaluation of all strain parameters from a single volumetric data set, effectively reducing the time needed for assessment [13-15]. This technique has the potential to perform a more reliable and complete assessment in patients treated with chemotherapy. However, it is still poorly studied in this population.

The aim of this study was to investigate the usefulness of 3D STE in evaluating left ventricle mechanics and its relation to CTRCD in a population of breast cancer patients submitted to anthracycline treatment.

\section{Materials and methods}

\section{Study design}

This was a sub-analysis of a single-center prospective observational study of patients with breast cancer undergoing chemotherapy with anthracyclines between August 2011 and August 2018. Patients were included after referral by the center's Oncology department for echocardiographic assessment, which included standard parameters and 2D global longitudinal strain (GLS). At the discretion of the echocardiographer, 3D echocardiography was performed in a subgroup of patients. Patients with 3D STE data available before the initiation of chemotherapy and during/after treatment were selected for this analysis. To avoid confounding factors that may affect left ventricle function, those with previous history of cardiovascular risk factors (arterial hypertension, diabetes mellitus, and chronic kidney disease), heart disease (coronary artery disease, cardiomyopathy, arrhythmias, prosthetic heart valves, implantable cardiac 
devices, or congenital heart disease), cardiac symptoms/ signs, cardiac medications, or significant pathological findings on the baseline echocardiography (LVEF $<54 \%$, left ventricle end-diastolic diameter $>32 \mathrm{~mm} / \mathrm{m}^{2}$, left ventricle septal or posterior wall thickness $>9 \mathrm{~mm}$, more than mild valvular heart disease, or wall motion abnormalities) were excluded. Demographic data, anticancer therapy and echocardiographic parameters were recorded.

\section{Echocardiographic acquisition and processing}

Initial and follow-up echocardiographic assessments were performed according to the clinical indications established by the oncologist. All echocardiograms were performed by one experienced cardiologist, unblinded to the previous exams. Echocardiographic parameters were determined according to the American Society of Echocardiography/ European Association of Cardiovascular Imaging's recommendations [7, 16-18]. Vivid 95 or Vivid 9 ultrasound systems (GE Healthcare) were used to acquire parasternal longand short-axis views, as well as apical 4-, 2-, and 3-chamber views. Left and right atrial volumes were assessed by the biplane and single-plane methods of disks, respectively. LVEF was measured using the biplane Simpson's method. A 3D volumetric transducer was used for real-time full-volume data acquisition in apical view of the left ventricle over four consecutive cardiac cycles during a single breath hold.

Data sets were analyzed on a workstation (EchoPAC BT12 workstation, GE Healthcare) equipped with a commercially available software for offline quantification of $2 \mathrm{D}$ longitudinal strain, 3D volumes, and 3D strain using the speckle-tracking technique with semi-automatic tracing of the endo- and epicardium following manual delineation of the mitral valve edges and apex. Whenever the tracing was not deemed to be correct, it was manually changed by the operator. 2D and 3D global strain parameters were calculated as averages of the regional values from the 18 or 17 myocardial segments, respectively. Rejected segments were excluded from the calculation of global strain values. If more than three segments were rejected, global strain values were not calculated.

\section{Definitions}

CTRCD was defined as an absolute decrease in 2D or 3D LVEF $>10 \%$ to a value $<54 \%$ or a relative decrease in $2 \mathrm{D}$ GLS $>15 \%[7,12]$.

Abnormal 3D global strain parameters were defined according to vendor-specific reference values for women with the following lower limits of normal: $-15 \%$ for 3D GLS, $-14 \%$ for 3D global circumferential strain (GCS), 40\% for 3D global radial strain (GRS), and $-27 \%$ for 3D global area strain (GAS) [19]. All strain values are interpreted on their absolute magnitude, such that more negative values represent better deformation for longitudinal, circumferential, and area strain and more positive values represent better deformation for radial strain. Percentage variation of strain values refers to the absolute value of the strain parameters, such that a negative variation corresponds to a worsening deformation and a positive variation to an increasing deformation.

Cumulative doses of each anthracycline were converted to doxorubicin equivalents by considering drug potency [20].

\section{Statistical analysis}

Continuous variables were expressed as mean and standard deviation when they followed a normal distribution, and as median and interquartile range otherwise. Qualitative variables were expressed as frequency and percentage. Changes in 2D and 3D echocardiographic parameters were analyzed by comparing baseline with follow-up echocardiograms. When more than one follow-up echocardiogram had been performed, the values corresponding to the greatest variation in the study variables were used. Incidence of CTRCD was assumed if at least one echocardiogram presented the above-mentioned criteria when compared to the baseline assessment. Continuous variables were compared using the Student's $t$ test and Wilcoxon sign-rank test, as appropriate, while categorical variables were compared using the Chisquare test. Correlation between echocardiographic variables and cumulative anthracycline dose was analyzed with the Pearson's and Spearman's correlation coefficients, as appropriate. Logistic regression analysis was used to determine predictors of CTRCD, with the incidence of CTRCD during follow-up as the dependent variable and including demographic characteristics, baseline echocardiographic parameters, anticancer therapy, and variations of 3D strain values as independent variables. Variables with $p$ value $<0.05$ on the univariate analysis were included in the multivariate model. Receiver operating curve analysis was used to assess the discrimination of variations of 3D strain values for predicting CTRCD. The optimum cutoff value for discriminating CTRCD was determined using the maximum likelihood ratio. A two-tailed $p$ value $<0.05$ was considered to be statistically significant. Statistical analysis was performed with the software package SPSS, version 23.0 (IBM Corp, Armonk, NY).

\section{Results}

\section{Baseline characteristics}

After excluding six patients (three for atrial fibrillation, one for arterial hypertension, one for coronary artery 
disease, and one for abnormal LVEF at baseline), a total of 105 women were assessed during a mean follow-up of $12.1 \pm 11.5$ months. Mean age at first echocardiogram was $53.8 \pm 12.5$ years (range $27-77$ years). The mean cumulative anthracycline dose was $259.6 \pm 63.3 \mathrm{mg} / \mathrm{m}^{2} .52 .4 \%$ of patients were also submitted to human epidermal growth factor receptor 2 (HER2) inhibitor therapy and $77.2 \%$ to radiotherapy. All patients underwent at least two echocardiographic studies, with an average of 3.8 exams per

Table 1 Baseline characteristics

\begin{tabular}{ll}
\hline Age (years) & $53.8 \pm 12.5$ \\
Cumulative anthracycline dose $\left(\mathrm{mg} / \mathrm{m}^{2}\right)$ & $259.6 \pm 63.3$ \\
HER2 inhibitor therapy, $n(\%)$ & $55(52.4)$ \\
Radiotherapy, $n(\%)$ & $87(77.2)$ \\
LV end-diastolic diameter $(\mathrm{mm})$ & $49.7 \pm 5.5$ \\
LV end-systolic diameter $(\mathrm{mm})$ & $29.6 \pm 6.0$ \\
Fractional shortening $(\%)$ & $40.8 \pm 7.4$ \\
LV septal thickness $(\mathrm{mm})$ & $8.3 \pm 2.1$ \\
LV posterior wall thickness $(\mathrm{mm})$ & $7.8 \pm 1.4$ \\
Aortic root diameter (mm) & $28.4 \pm 3.7$ \\
LA diameter (mm) & $35.2 \pm 4.6$ \\
TAPSE (mm) & $22.5 \pm 3.1$ \\
Tricuspid $s^{\prime}(\mathrm{cm} / \mathrm{s})$ & $12.9 \pm 3.0$ \\
Transtricuspid pressure gradient $(\mathrm{mmHg})$ & $21.0 \pm 7.4$ \\
\hline
\end{tabular}

$L A$ left atrium, $L V$ left ventricle, TAPSE tricuspid annular plane systolic excursion patient at a mean interval of 123 days. Baseline characteristics, including standard baseline echocardiographic parameters, are shown in Table 1.

\section{Incidence of cancer therapeutics-related cardiac dysfunction}

CTRCD occurred in 24 (22.9\%) patients during follow-up. Most patients $(n=14)$ were diagnosed according to the GLS criterion. Cumulative anthracycline dose predicted CTRCD (odds ratio [OR] $1.08895 \%$ confidence interval [CI] 1.001-1.022 $p=0.033)$. Six (5.7\%) patients dropped out of anthracycline therapy because of cardiac dysfunction (mean LVEF during chemotherapy $43.6 \% \pm 9.8 \%$ ).

\section{Standard echocardiographic follow-up}

Table 2 displays the standard echocardiographic parameters for the assessment of CTRCD before and during chemotherapy. LVEF, either 2D- or 3D-derived, as well as 2D GLS showed a significant reduction during anthracycline treatment (all $p<0.001)$. Both left and right atrial volumes increased (all $p<0.001)$ and mitral deceleration time decreased $(p=0.042)$ during chemotherapy.
Table 2 Echocardiographic parameters before and during chemotherapy

\begin{tabular}{|c|c|c|c|}
\hline & Baseline & During chemotherapy & $p$ value \\
\hline \multicolumn{4}{|l|}{ 2D parameters } \\
\hline LV end-diastolic volume (ml) & $92.3 \pm 23.0$ & $99.6 \pm 27.3$ & 0.043 \\
\hline LV end-systolic volume (ml) & $31.7 \pm 11.7$ & $42.0 \pm 13.4$ & 0.021 \\
\hline $\operatorname{LVEF}(\%)$ & $65.6 \pm 8.0$ & $57.8 \pm 11.0$ & $<0.001$ \\
\hline LV stroke volume (ml) & $60.6 \pm 3.0$ & $57.6 \pm 1.8$ & 0.014 \\
\hline LV cardiac output (1/min) & $5.2 \pm 1.6$ & $4.6 \pm 2.0$ & 0.011 \\
\hline LA volume (ml) & $44.1 \pm 12.7$ & $54.0 \pm 13.1$ & $<0.001$ \\
\hline RA volume (ml) & $27.4 \pm 10.0$ & $36.3 \pm 10.5$ & $<0.001$ \\
\hline Transmitral $E / A$ ratio & $1.2 \pm 0.1$ & $1.1 \pm 0.5$ & 0.586 \\
\hline Transmitral declaration time (ms) & $200.2 \pm 46.2$ & $188.1 \pm 48.0$ & 0.042 \\
\hline Mitral $E / e^{\prime}$ ratio & $7.9 \pm 3.7$ & $7.7 \pm 3.4$ & 0.682 \\
\hline \multicolumn{4}{|l|}{ 2D strain parameter } \\
\hline Global longitudinal strain (\%) & $-21.1 \pm 3.0$ & $-18.8 \pm 3.1$ & $<0.001$ \\
\hline \multicolumn{4}{|l|}{$3 \mathrm{D}$ volumetric parameters } \\
\hline LV end-diastolic volume (ml) & $84.0 \pm 17.0$ & $89.9 \pm 30.8$ & 0.156 \\
\hline LV end-systolic volume (ml) & $32.3 \pm 9.4$ & $41.0 \pm 26.6$ & 0.002 \\
\hline $\operatorname{LVEF}(\%)$ & $61.5 \pm 6.0$ & $54.4 \pm 8.8$ & $<0.001$ \\
\hline LV stroke volume (ml) & $51.7 \pm 26.9$ & $48.9 \pm 12.2$ & 0.010 \\
\hline LV cardiac output ( $1 / \mathrm{min})$ & $4.3 \pm 1.7$ & $3.9 \pm 1.4$ & $<0.001$ \\
\hline
\end{tabular}

$2 D$ two-dimensional, $3 D$ three-dimensional, $L A$ left atrium, $L V$ left ventricle, $L V E F$ left ventricle ejection fraction, $R A$ right atrium 


\section{Three-dimensional global strain analysis}

Values of 3D GLS, 3D GCS, 3D GRS, and 3D GAS all showed a significant worsening during anthracycline treatment (all $p<0.001$ ). Percentage variation was numerically greater for 3D GCS and 3D GRS (Table 3). A high percentage of the total study population presented 3D global strain values below the limits of normal during chemotherapy: $81.4 \%$ for GLS, $73.1 \%$ for GCS, $83.6 \%$ for GRS, and $88.6 \%$ for GAS (Fig. 1). Cumulative anthracycline dose was correlated with 3D GCS $(r=0.349, p=0.026)$ and 3D GRS $(r=-0.328, p=0.034)$. 3D GLS $(-9.7 \pm 3.6$ vs. $-12.1 \pm 3.9, p=0.021)$ and $3 \mathrm{D}$ GRS $(24.2 \pm 11.5$ vs. $31.6 \pm 12.5, p=0.038$ ) were lower in patients who were treated with HER2 inhibitor therapy (Online Resource 1).

\section{Three-dimensional regional strain analysis}

Overall, regional 3D strain could be determined in $94.0 \%$ of the segments analyzed, with lower success rates in the basal inferior (79.3\%) and basal inferolateral (82.1\%) walls. 2D regional strain analysis showed a greater feasibility, with adequate assessment of $96.1 \%$ of segments $(p<0.001)$. There was no difference in the ability to analyze regional 3D strain before and during chemotherapy. When comparing echocardiograms before and during chemotherapy, there was a significantly impaired 3D longitudinal strain in $13 \mathrm{seg}$ ments, circumferential strain in 7 segments, radial strain in 14 segments, and area strain in 11 out of 17 segments (Figs. 1,2). Overall, the anterior, inferior, and septal walls were most consistently affected, while the lateral wall and apex were relatively spared.

\section{Relationship of three-dimensional strain and cancer therapeutics-related cardiac dysfunction}

There was no difference in 3D global strain values in patients with and without CTRCD. On the other hand, 3D GCS variation $(-38.2 \%$ vs. $-24.9 \% p=0.020)$ and 3D GRS variation $(-41.9 \%$ vs. $-27.5 \% p=0.024)$ were significantly greater in patients with diagnosed CTRCD (Fig. 3). Atrial volumes and diastolic function parameters were not correlated with 3D STE values.

Logistic regression analysis showed that variations of 3D GRS and 3D GCS were associated with a higher incidence of CTRCD. In the multivariate model, the variation of 3D GRS remained the only independent predictor of CTRCD (Table 4).

The receiver operating curve analysis showed a good discrimination of variations of 3D GCS and 3D GRS in predicting CTRCD with areas under the curve of $0.748(p=0.033)$
Table 3 3D-derived strain parameters before and during chemotherapy

\begin{tabular}{lcccc}
\hline & Baseline & During chemotherapy & $p$ value & Variation \\
\hline 3D global longitudinal strain (\%) & $-15.6 \pm 3.4$ & $-10.9 \pm 4.1$ & $<0.001$ & $-25.5 \pm 22.7$ \\
3D global circumferential strain (\%) & $-14.0 \pm 4.0$ & $-11.0 \pm 5.0$ & $<0.001$ & $-29.3 \pm 20.0$ \\
3D global radial strain (\%) & $42.0 \pm 17.0$ & $28.5 \pm 17.5$ & $<0.001$ & $-29.4 \pm 24.0$ \\
3D global area strain (\%) & $-27.0 \pm 8.5$ & $-20.0 \pm 9.0$ & $<0.001$ & $-25.0 \pm 23.1$ \\
\hline
\end{tabular}

$3 D$ three-dimensional

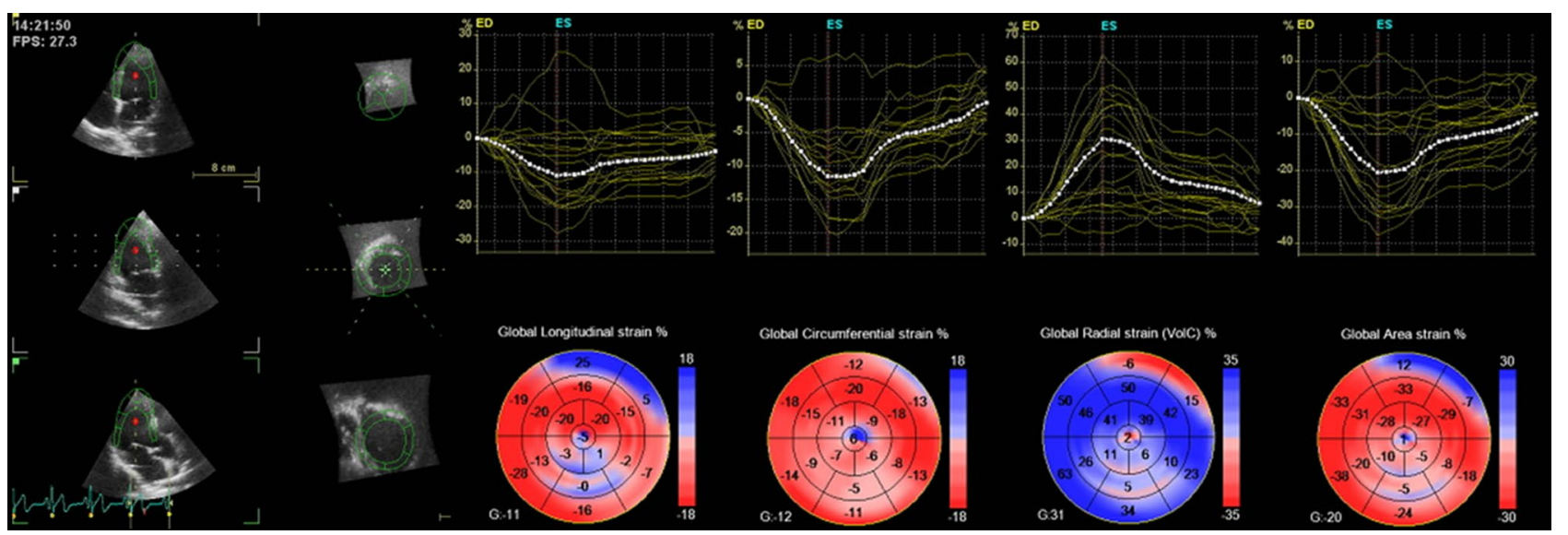

Fig. 1 Representative example of 3D speckle-tracking strain analysis in a breast cancer patient during chemotherapy with anthracyclines showing semi-automatic tracking of the endo- and epicardium, global longitudinal, circumferential, radial and area strain values $(G)$, as well as corresponding regional values presented as a color-coded 17-segment bull's eye plot. Note that all strain parameters are below the lower limits of normal and that anterior, inferolateral, and septal walls are most often affected 
Fig. 2 3D regional longitudinal (3D LS), circumferential (3D CS), radial (3D RS), and area (3D AS) strain. Numbers represent $p$ values of the Student's $t$ test comparing echocardiograms before and during chemotherapy for each segment
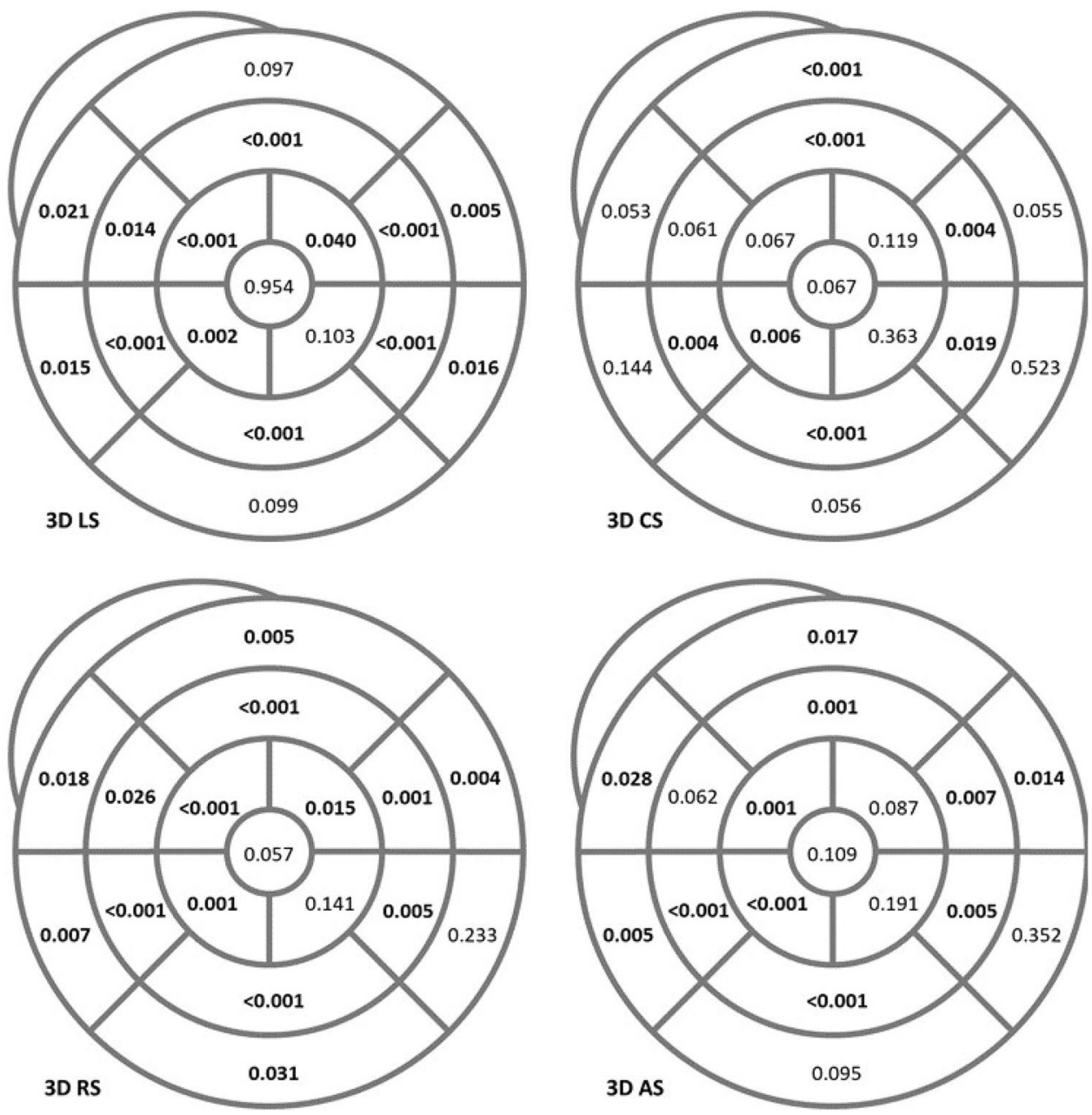

Fig. 3 3D global strain variation according to the presence or absence of CTRCD. Dark grey bars represent patients without CTRCD. Light grey bars represent patients with CTRCD. Significant differences in variation were seen for 3D GCS and 3D GRS. 3D three-dimensional, $G A S$ global area strain, $G C S$ global circumferential strain; GLS global longitudinal strain; GRS global radial strain
$-50 \%$

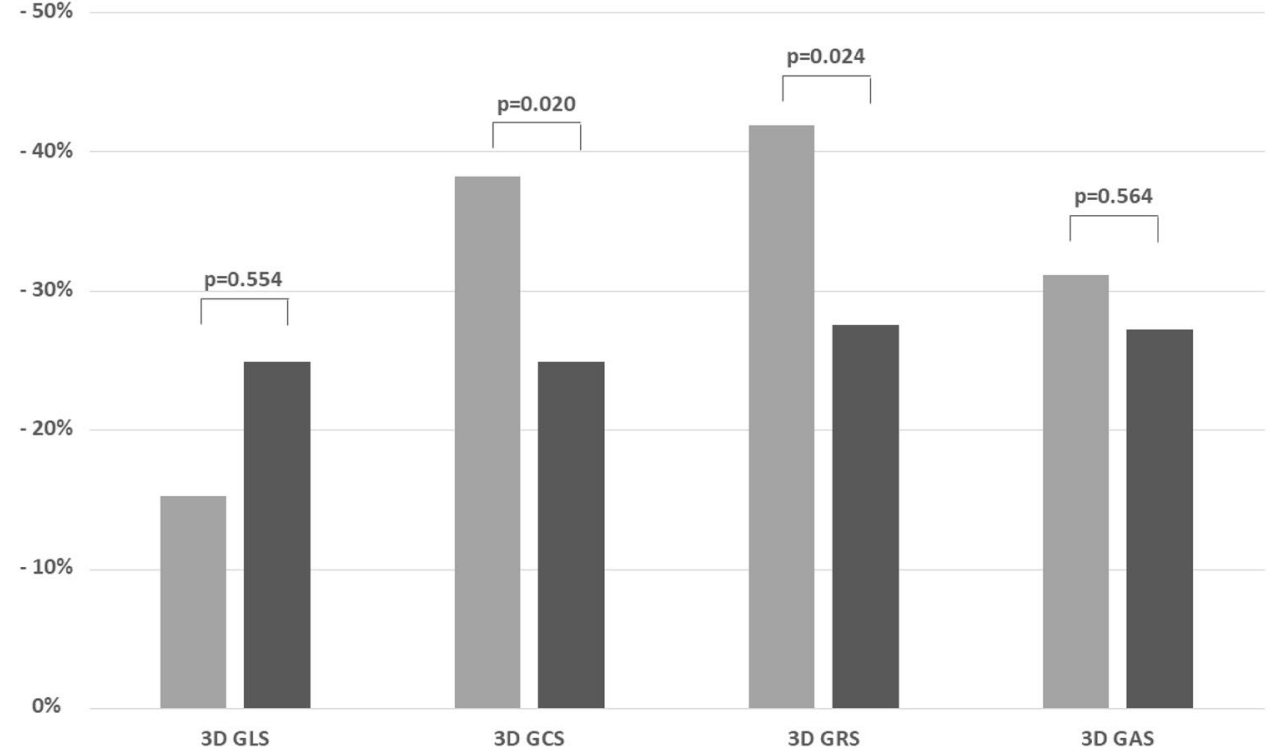

and $0.719(\mathrm{p}=0.047)$, with the optimal cutoff values being $-34.2 \%$ (sensitivity $63.6 \%$, specificity $73.3 \%$, and likelihood ratio 2.38 ) and $-34.4 \%$ (sensitivity $72.7 \%$, specificity $68.7 \%$, and likelihood ratio 2.32), respectively (Fig. 4). According to these cutoffs, $26.9 \%$ of this cohort showed significant variations of 3D GCS and $48.2 \%$ significant variations of 3D GRS. 
Table 4 Logistic regression analysis for predictors of cancer therapeutics-related cardiac dysfunction

\begin{tabular}{|c|c|c|c|c|}
\hline & \multicolumn{2}{|l|}{ Univariate analysis } & \multicolumn{2}{|c|}{ Multivariate analysis } \\
\hline & OR $(95 \% \mathrm{CI})$ & $p$ value & OR $(95 \% \mathrm{CI})$ & $p$ value \\
\hline Age & $1.01(0.97-1.05)$ & 0.556 & - & \\
\hline Anthracycline cumulative dose & $1.09(1.00-1.02)$ & $\mathbf{0 . 0 3 3}$ & $0.99(0.95-1.04)$ & 0.761 \\
\hline Radiotherapy & $3.34(0.38-29.71)$ & 0.275 & - & \\
\hline HER2 inhibitor therapy & $0.57(0.21-1.60)$ & 0.287 & - & \\
\hline Fractional shortening & $0.95(0.89-1.02)$ & 0.149 & - & \\
\hline Mitral E/e' ratio & $1.08(0.93-1.25)$ & 0.314 & - & \\
\hline 3D GLS variation & $0.53(0.06-4.33)$ & 0.550 & - & \\
\hline 3D GCS variation & $1.97(1.63-2.38)$ & 0.036 & $1.38(0.01-5.69)$ & 0.952 \\
\hline 3D GRS variation & $5.95(1.29-2.76)$ & 0.041 & $1.39(1.13-2.18)$ & 0.016 \\
\hline 3D GAS variation & $4.51(0.03-6.18)$ & 0.549 & - & - \\
\hline
\end{tabular}

$3 D$ three-dimensional, $C I$ confidence interval, $G A S$ global area strain, GCS global circumferential strain, $G L S$ global longitudinal strain, GRS global radial strain, $O R$ odds ratio

$p$ values $<0.05$ are highlighted in bold

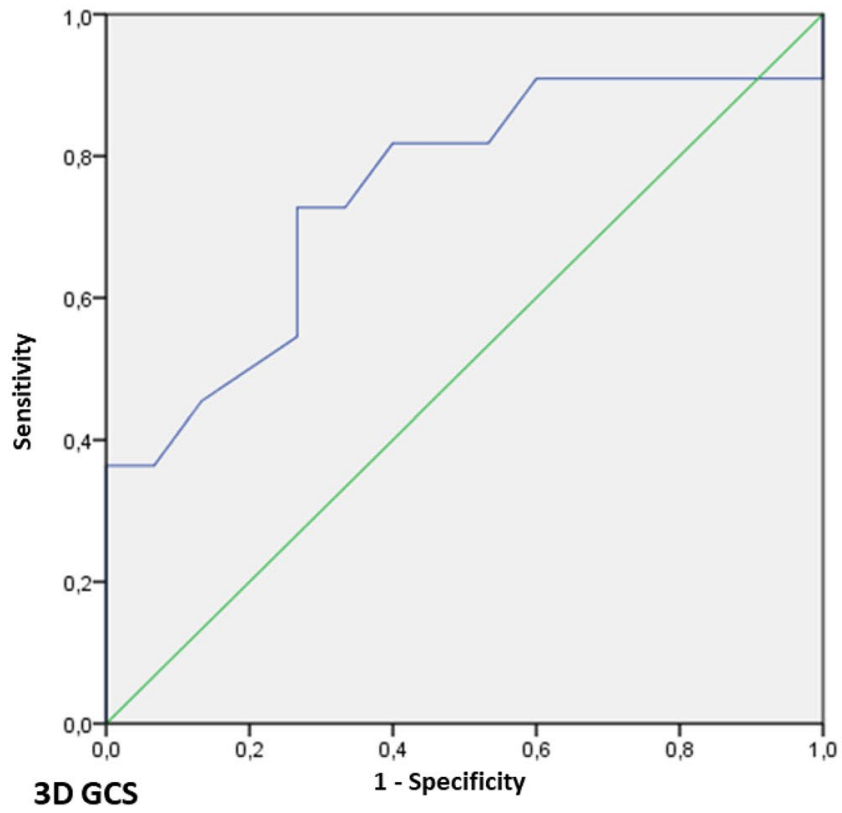

Fig. 4 Receiver operating curves of variations of 3D GCS and 3D GRS for prediction of CTRCD, with areas under de curve of 0.748 $(p=0.033)$ and $0.719(p=0.047)$, with the optimal cutoff values

Among these, $63.6 \%$ and $58.3 \%$ went on to develop CTRCD, respectively. These variations were observed a median of 45 days and 23 days before the diagnosis of CTRCD.

\section{Discussion}

CTRCD induced by anthracyclines is a growing problem that affects the outcome of cancer patients [2]. The reversibility of cardiac dysfunction is inversely related to the time since the start of chemotherapy $[21,22]$. As such, there has

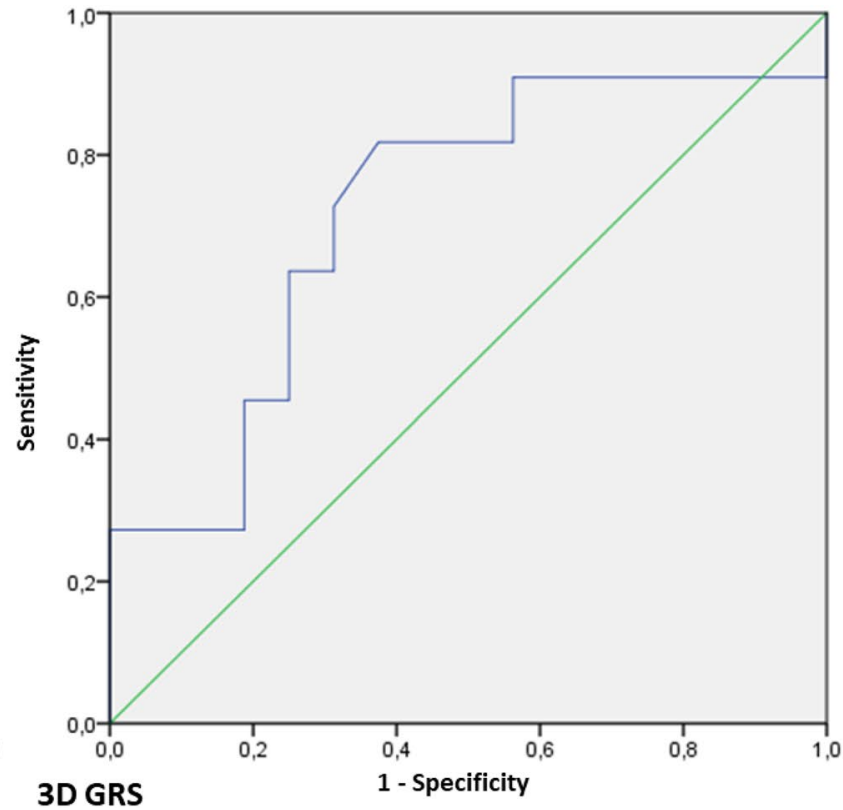

being $-34.2 \%$ (likelihood ratio 2.38 ) and $-34.4 \%$ (likelihood ratio 2.32), respectively. $3 D$ three-dimensional; $G C S$ global circumferential strain, GRS global radial strain

been a continuous search for the optimal screening technique to diagnose subclinical dysfunction to allow for timely initiation of neurohumoral therapy and prevent the progression to overt heart failure. 2D STE has proved to detect early impairment of myocardial systolic function before LVEF decreases [11]. 2D GLS is recommended over other 2D strain parameters [12] because of concerns about the reproducibility of measurements of 2D GRS and 2D GCS [11]. Besides, the evaluation of all strain parameters by 2D STE is time-consuming and not adequate for clinical practice. 
3D STE assessment of myocardial deformation has several advantages, such as the ability to track out-of-plane motion of speckles, higher reproducibility, and simultaneous evaluation of all strain parameters [13-15].

In the present study, we hypothesized that 3D STE would be a better technique for global and regional evaluation of left ventricle mechanics and CTRCD in breast cancer patients submitted to anthracycline chemotherapy. The main findings of our study were: (1) CTRCD is a common occurrence in this high-risk population; (2) a significant decrease in all 3D strain parameters was observed, with a high percentage of patients showing abnormal values during chemotherapy; (3) anterior, inferior, and septal walls were most commonly affected; and (4) percentage variations of 3D GCS and 3D GRS were good early predictors of CTRCD.

\section{Incidence of cancer therapeutics-related cardiac dysfunction}

We report an incidence of CTRCD that is higher than in the previous studies for the described cumulative dose [12]. As the definition of CTRCD changes towards a more sensitive one, it is expected that its incidence will be higher than in older studies employing LVEF instead of 2D GLS. Another factor that probably contributed to this incidence is the high percentage of patients treated with HER2 inhibitor therapy, which is known to have an additive effect to anthracyclines [23]. Cumulative anthracycline dose was a determinant of CTRCD, as described previously [12].

\section{Worsening of three-dimensional global strain during chemotherapy}

The use of 3D STE in this population demonstrates that left ventricle mechanics are affected in all strain components during chemotherapy. This is concordant with the evidence obtained with 2D STE analyzing 2D GLS, 2D GCS, and 2D GRS [24], as well as with more recent reports of 3D STE [25-30]. 3D GCS and 3D GRS had a more pronounced variation than 3D GLS and 3D GAS. In fact, the previous reports employing 2D STE already hinted at a possibility of a greater affliction of 2D GRS [31-35] and 2D GCS [33, 36,37 , although results were highly variable [24]. Studies using 3D STE also showed inconsistent results, with greater affliction of 3D GCS and 3D GAS [27], 3D GRS and 3D GAS [26], 3D GLS and 3D GRS [38], 3D GRS [25, 39], or 3D GAS [40].

Interestingly, baseline 3D strain parameters were around the lower limits of normal. Abnormal values of 2D strain parameters in cancer patients before treatment initiation have previously been reported [41], suggesting a direct effect of cancer on cardiac mechanics.
Cumulative anthracycline dose and HER2 inhibitor therapy were related to lower values of 3D strain parameters. This correlation was previously described for cumulative anthracycline dose using 3D STE [25, 40, 42], but not for HER2 inhibitor therapy.

\section{Regional pattern of cancer therapeutics-related cardiac dysfunction}

The anterior, inferior, and septal walls were more commonly affected in this cohort, possibly indicating a regional heterogeneity in the development of systolic dysfunction. Previous authors have published similar patterns, mainly affecting the septum, using 2D [32, 43-45] and 3D [39] STE, cardiac magnetic resonance [46, 47], and visual evaluation of wall motion abnormalities [48]. The explanation for this regional pattern is currently unclear, but could lie in shear stress forces acting differently in relation to left ventricle geometry [49], the increased exposure to anthracyclines in regions of terminal circulation [50], or the differential local activation of signal transduction pathways of fibrosis or apoptosis [51]. While this needs confirmation in further studies, the recognition of this regional pattern may prove useful when screening for CTRCD.

\section{Prediction of cardiac dysfunction by three-dimensional strain}

2D STE is a sensitive marker of CTRCD, with a number of studies showing a decrease in 2D global strain parameters before an abnormal LVEF was evident [11, 52]. Furthermore, a decrease in 2D GLS predicts the subsequent development of cardiac dysfunction measured by LVEF [11]. On the other hand, results on 2D GRS predicting CTRCD are less consistent [53-55] and inexistent for 2D GCS.

Evidence that 3D STE could predict subsequent changes in 2D LVEF was first given by Mornoş et al. [25]. Zhang and colleagues then proved that this prediction was independent of 2D strain values [29]. To date, only two studies have shown that decreases in 3D strain can occur at a timepoint, when both 2D strain and LVEF were unchanged $[28,40]$. While this could be an important finding showing added value of 3D over 2D STE, the authors failed to explore its relation to subsequent decreases of 2D GLS.

Similarly, our results show a much higher proportion of patients with abnormal 3D strain values than the percentage with CTRCD. This suggests the presence of subclinical myocardial dysfunction, even in patients who do not go on to develop CTRCD. 3D STE is, therefore, a more sensitive method to screen for cardiac dysfunction. This is the first study, where 3D STE, namely, 3D GCS 
and 3D GRS variations, showed good predictive ability for cardiotoxicity diagnosed by an up-to-date definition with 2D/3D LVEF and 2D GLS as criteria. In particular, 3D GRS was the only independent predictor among other known determinants of cardiac dysfunction. The superiority of these parameters for predicting LVEF has also been described by other authors [29, 38]. Although the early detection of myocardial changes is conceptually important, the real value of these changes lies in their ability to prognosticate a subsequent LVEF decrease. Importantly, our results are also the first to show that variations of 3D GCS and 3D GRS preceded the diagnosis of CTRCD by a median of 45 and 23 days, establishing this method as an important tool for the follow-up of patients submitted to anthracycline therapy. We provide cutoff values of strain variation for detecting subsequent CTRCD $(-34.2 \%$ for 3D GCS and $-34.4 \%$ for 3D GRS) that still need external validation, but are a starting point for further studies. This could allow closer monitoring of this population and even earlier initiation of cardioprotective therapies before the development of left ventricle dysfunction. Future recommendations could rely on screening of a combination of 3D STE and cardiac biomarkers. A possible algorithm could be repeating the echocardiogram 2-4 weeks after a significant variation in 3D STE and starting beta-blockers and angiotensin converting enzyme inhibitors if cardiac biomarkers are also increased.

\section{Feasibility of three-dimensional strain analysis}

Low feasibility, mainly due to poor acoustic window, has been described for 3D STE when compared with 2D STE $[19,25,27]$. In our study, 3D echocardiography was performed at the discretion of the echocardiographer, which means that a significant number of patients might have been excluded due to insufficient image quality. However, more recent reports have described excellent feasibility, as well as lower inter- and intra-observer variability of 3D STE compared to 2D STE [29]. Concerns have emerged regarding the adverse effects of cancer therapeutics (radiotherapy, mastectomy, and breast prothesis) on the ability to perform 3D STE [27]. We did not find any significant differences in feasibility comparing echocardiograms before and during chemotherapy.

On a regional level, even though we found that the feasibility of 3D STE was lower than that of 2D STE, it was still excellent (94.0\%). The basal inferior and basal inferolateral walls were the segments with the least feasibility. This is congruent with the previous studies, where these segments are the most challenging to track because of their active excursion and position in the far field in apical data sets $[19,27]$.

\section{Study limitations}

The main limitation of this study is the absence of clinical events as endpoints, which precludes their correlation to echocardiographic parameters. It would be important to evaluate the prognostic value of worsening 3D strain parameters. Although 3D strain parameters have already been associated with clinical events in other contexts [56], this relationship has not been established for cancer patients. Equally, improved outcomes with earlier initiation of cardioprotective therapies guided by 3D strain still needs to be proven. An intrinsic limitation of any study that addresses the natural history of CTRCD based on echocardiographic parameters is that the variables analyzed are measures of left ventricle contractile or systolic function and overlap between them may exist. Notwithstanding, our findings have important clinical implications, since they could allow earlier diagnosis of cardiac dysfunction, more targeted monitoring and timely initiation of cardioprotective treatment. Furthermore, the fact that echocardiographic exams were performed at the discretion of the oncologist and the relatively short follow-up of this cohort may have contributed to an underestimation of CTRCD. In patients with fewer echocardiograms or shorter follow-up, exposure to anthracyclines may not have reached a sufficiently high cumulative dose to produce a significant decrease in LVEF or 2D GLS. Nevertheless, it is clear in this cohort that abnormal 3D strain parameters were much more common than CTRCD and that 3D STE predicted the subsequent diagnosis of CTRCD. An additional limitation is the fact that a significant proportion of patients also received HER2 inhibitor therapy and/or radiotherapy, such that it is difficult to assess the sole contribution of anthracyclines to cardiac dysfunction. The results cannot then be generalized to those under anthracycline therapy alone. Finally, the reference values for 3D strain are not yet fully established. We used vendor-specific reference values described in a large cohort of healthy subjects [19] and inter-vendor inconsistencies of 3D strain parameters are well documented [57]. As such, caution should be exerted against direct comparison of results obtained by different scanners. However, using the percentage variation of 3D strain values as the main subject of our analysis, we intended to produce evidence that would be practical in as many settings as possible and potentially applicable to other vendors.

\section{Conclusion}

Left ventricle contractility, as assessed by 3D STE, worsened during anthracycline therapy for breast cancer, with predominant involvement of septal, anterior, and inferior walls. Percentage variations of 3D GCS and 3D GRS were predictive of subsequent CTRCD, and thus can be considered an earlier 
sign of CTRCD, with added value over the currently recommended 2D or 3D LVEF and 2D GLS. Routine application of this technique should be considered in cancer patients treated with chemotherapy to offer targeted monitoring and timely initiation of cardioprotective treatment.

\section{Compliance with ethical standards}

Conflicts of interest The authors declare that they have no conflict of interest.

Ethical standards This study has been performed in accordance with the ethical standards laid down in the 1964 Declaration of Helsinki and its later amendments.

Informed consent Informed consent was obtained from all individual participants included in the study.

\section{References}

1. Swain SM, Whaley FS, Ewer MS (2003) Congestive heart failure in patients treated with doxorubicin: a retrospective analysis of three trials. Cancer 97:2869-2879. https://doi.org/10.1002/ cncr. 11407

2. Doyle JJ, Neugut AI, Jacobson JS, Grann VR, Hershman DL (2005) Chemotherapy and cardiotoxicity in older breast cancer patients: a population-based study. J Clin Oncol 23:8597-8605. https://doi.org/10.1200/JCO.2005.02.5841

3. Ramos A, Meyer RA, Korfhagen J, Wong KY, Kaplan S (1976) Echocardiographic evaluation of adriamycin cardiotoxicity in children. Cancer Treat Rep 60:1281-1284

4. Lenzhofer R, Dudczak R, Gumhold G, Graninger W, Moser K, Spitzy KH (1983) Noninvasive methods for the early detection of doxorubicin-induced cardiomyopathy. J Cancer Res Clin Oncol 106:136-142

5. Ewer MS, Ali MK, Mackay B, Wallace S, Valdivieso M, Legha SS, Benjamin RS, Haynie TP (1984) A comparison of cardiac biopsy grades and ejection fraction estimations in patients receiving adriamycin. J Clin Oncol 2:112-117. https://doi.org/10.1200/ JCO.1984.2.2.112

6. Thavendiranathan P, Grant AD, Negishi T, Plana JC, Popovic ZB, Marwick TH (2013) Reproducibility of echocardiographic techniques for sequential assessment of left ventricular ejection fraction and volumes: application to patients undergoing cancer chemotherapy. J Am Coll Cardiol 61:77-84. https://doi.org/10.1016/j. jacc.2012.09.035

7. Lang RM, Badano LP, Mor-Avi V, Afilalo J, Armstrong A, Ernande L, Flachskampf FA, Foster E, Goldstein SA, Kuznetsova T, Lancellotti P, Muraru D, Picard MH, Rietzschel ER, Rudski L, Spencer KT, Tsang W, Voigt JU (2015) Recommendations for cardiac chamber quantification by echocardiography in adults: an update from the American Society of Echocardiography and the European Association of Cardiovascular Imaging. Eur Heart J Cardiovasc Imaging 16:233-270. https://doi.org/10.1093/ehjci/ jev014

8. Armstrong GT, Plana JC, Zhang N, Srivastava D, Green DM, Ness KK, Daniel Donovan F, Metzger ML, Arevalo A, Durand JB, Joshi V, Hudson MM, Robison LL, Flamm SD (2012) Screening adult survivors of childhood cancer for cardiomyopathy: comparison of echocardiography and cardiac magnetic resonance imaging. J Clin Oncol 30:2876-2884. https://doi.org/10.1200/ JCO.2011.40.3584

9. Cardinale D, Sandri MT, Martinoni A, Borghini E, Civelli M, Lamantia G, Cinieri S, Martinelli G, Fiorentini C, Cipolla CM (2002) Myocardial injury revealed by plasma troponin I in breast cancer treated with high-dose chemotherapy. Ann Oncol 13:710 715. https://doi.org/10.1093/annonc/mdf170

10. Morris PG, Dickler M, McArthur HL, Traina T, Sugarman S, Lin N, Moy B, Come S, Godfrey L, Nulsen B, Chen C, Steingart R, Rugo H, Norton L, Winer E, Hudis CA, Dang CT (2009) Dosedense adjuvant doxorubicin and cyclophosphamide is not associated with frequent short-term changes in left ventricular ejection fraction. J Clin Oncol 27:6117-6123. https://doi.org/10.1200/ JCO.2008.20.2952

11. Thavendiranathan P, Poulin F, Lim KD, Plana JC, Woo A, Marwick TH (2014) Use of myocardial strain imaging by echocardiography for the early detection of cardiotoxicity in patients during and after cancer chemotherapy: a systematic review. J Am Coll Cardiol 63:2751-2768. https://doi.org/10.1016/j.jacc.2014.01.073

12. Zamorano JL, Lancellotti P, Rodriguez Munoz D, Aboyans V, Asteggiano R, Galderisi M, Habib G, Lenihan DJ, Lip GYH, Lyon AR, Lopez Fernandez T, Mohty D, Piepoli MF, Tamargo J, Torbicki A, Suter TM, Group ESCSD (2016) 2016 ESC position paper on cancer treatments and cardiovascular toxicity developed under the auspices of the esc committee for practice guidelines: the task force for cancer treatments and cardiovascular toxicity of the european society of cardiology. Eur Heart J. 37:2768-2801. https://doi.org/10.1093/eurheartj/ehw211

13. de Isla PL, Balcones DV, Fernandez-Golfin C, Marcos-Alberca P, Almeria C, Rodrigo JL, Macaya C, Zamorano J (2009) Threedimensional-wall motion tracking: a new and faster tool for myocardial strain assessment: comparison with two-dimensional-wall motion tracking. J Am Soc Echocardiogr. 22:325-330. https://doi. org/10.1016/j.echo.2009.01.001

14. Mor-Avi V, Lang RM, Badano LP, Belohlavek M, Cardim NM, Derumeaux G, Galderisi M, Marwick T, Nagueh SF, Sengupta PP, Sicari R, Smiseth OA, Smulevitz B, Takeuchi M, Thomas JD, Vannan M, Voigt JU, Zamorano JL (2011) Current and evolving echocardiographic techniques for the quantitative evaluation of cardiac mechanics: ASE/EAE consensus statement on methodology and indications endorsed by the japanese society of echocardiography. J Am Soc Echocardiogr 24:277-313. https://doi. org/10.1016/j.echo.2011.01.015

15. Reant P, Barbot L, Touche C, Dijos M, Arsac F, Pillois X, Landelle M, Roudaut R, Lafitte S (2012) Evaluation of global left ventricular systolic function using three-dimensional echocardiography speckle-tracking strain parameters. J Am Soc Echocardiogr 25:68-79. https://doi.org/10.1016/j.echo.2011.10.009

16. Lang RM, Badano LP, Tsang W, Adams DH, Agricola E, Buck T, Faletra FF, Franke A, Hung J, de Isla LP, Kamp O, Kasprzak JD, Lancellotti P, Marwick TH, McCulloch ML, Monaghan MJ, Nihoyannopoulos P, Pandian NG, Pellikka PA, Pepi M, Roberson DA, Shernan SK, Shirali GS, Sugeng L, Cate FJT, Vannan MA, Zamorano JL, Zoghbi WA, American Society of E, European Association of E (2012) EAE/ASE recommendations for image acquisition and display using three-dimensional echocardiography. Eur Heart J Cardiovasc Imaging 13:1-46. https://doi. org/10.1093/ehjci/jer316

17. Voigt JU, Pedrizzetti G, Lysyansky P, Marwick TH, Houle H, Baumann R, Pedri S, Ito Y, Abe Y, Metz S, Song JH, Hamilton J, Sengupta PP, Kolias TJ, d'Hooge J, Aurigemma GP, Thomas JD, Badano LP (2015) Definitions for a common standard for 2D speckle tracking echocardiography: consensus document of the EACVI/ASE/industry task force to standardize deformation imaging. Eur Heart J Cardiovasc Imaging 16:1-11. https://doi. org/10.1093/ehjci/jeu184 
18. Nagueh SF, Smiseth OA, Appleton CP, Byrd BF 3rd, Dokainish H, Edvardsen T, Flachskampf FA, Gillebert TC, Klein AL, Lancellotti P, Marino P, Oh JK, Popescu BA, Waggoner AD, Houston T, Oslo N, Phoenix A, Nashville T, Hamilton OC, Uppsala S, Ghent T, Liege B, Cleveland O, Novara I, Rochester M, Bucharest R, St. Louis M (2016) Recommendations for the evaluation of left ventricular diastolic function by echocardiography: an update from the American Society of Echocardiography and the European Association of Cardiovascular Imaging. Eur Heart J Cardiovasc Imaging 17:1321-1360. https://doi.org/10.1093/ehjci/jew082

19. Muraru D, Cucchini U, Mihaila S, Miglioranza MH, Aruta P, Cavalli G, Cecchetto A, Padayattil-Jose S, Peluso D, Iliceto S, Badano LP (2014) Left ventricular myocardial strain by threedimensional speckle-tracking echocardiography in healthy subjects: reference values and analysis of their physiologic and technical determinants. J Am Soc Echocardiogr 27:858-871. https:// doi.org/10.1016/j.echo.2014.05.010 (e851)

20. Minotti G, Menna P, Salvatorelli E, Cairo G, Gianni L (2004) Anthracyclines: molecular advances and pharmacologic developments in antitumor activity and cardiotoxicity. Pharmacol Rev 56:185-229. https://doi.org/10.1124/pr.56.2.6

21. Cardinale D, Colombo A, Lamantia G, Colombo N, Civelli M, De Giacomi G, Rubino M, Veglia F, Fiorentini C, Cipolla CM (2010) Anthracycline-induced cardiomyopathy: clinical relevance and response to pharmacologic therapy. J Am Coll Cardiol 55:213220. https://doi.org/10.1016/j.jacc.2009.03.095

22. Ohtani K, Fujino T, Ide T, Funakoshi K, Sakamoto I, Hiasa KI, Higo T, Kamezaki K, Akashi K, Tsutsui H (2019) Recovery from left ventricular dysfunction was associated with the early introduction of heart failure medical treatment in cancer patients with anthracycline-induced cardiotoxicity. Clin Res Cardiol 108:600611. https://doi.org/10.1007/s00392-018-1386-0

23. Chen J, Long JB, Hurria A, Owusu C, Steingart RM, Gross CP (2012) Incidence of heart failure or cardiomyopathy after adjuvant trastuzumab therapy for breast cancer. J Am Coll Cardiol 60:2504-2512. https://doi.org/10.1016/j.jacc.2012.07.068

24. Bergamini C, Dolci G, Truong S, Zanolla L, Benfari G, Fiorio E, Rossi A, Ribichini FL (2019) Role of speckle tracking echocardiography in the evaluation of breast cancer patients undergoing chemotherapy: review and meta-analysis of the literature. Cardiovasc Toxicol. https://doi.org/10.1007/s12012-019-09523-y

25. Mornos C, Manolis AJ, Cozma D, Kouremenos N, Zacharopoulou I, Ionac A (2014) The value of left ventricular global longitudinal strain assessed by three-dimensional strain imaging in the early detection of anthracyclinemediated cardiotoxicity. Hellenic J Cardiol 55:235-244

26. Lorenzini C, Lamberti C, Aquilina M (2015) Speckle tracking analysis for early detection of cardiotoxicity in breast cancer patients. Comput Cardiol Nice France IEEE. 42:177-180. https ://doi.org/10.1109/CIC.2015.7408615

27. Santoro C, Arpino G, Esposito R, Lembo M, Paciolla I, Cardalesi C, de Simone G, Trimarco B, De Placido S, Galderisi M (2017) 2D and 3D strain for detection of subclinical anthracycline cardiotoxicity in breast cancer patients: a balance with feasibility. Eur Heart J Cardiovasc Imaging 18:930-936. https://doi.org/10.1093/ ehjci/jex033

28. Song FY, Shi J, Guo Y, Zhang CJ, Xu YC, Zhang QL, Shu XH, Cheng LL (2017) Assessment of biventricular systolic strain derived from the two-dimensional and three-dimensional speckle tracking echocardiography in lymphoma patients after anthracycline therapy. Int J Cardiovasc Imaging 33:857-868. https://doi. org/10.1007/s10554-017-1082-6

29. Zhang KW, Finkelman BS, Gulati G, Narayan HK, Upshaw J, Narayan V, Plappert T, Englefield V, Smith AM, Zhang C, Hundley WG, Ky B (2018) Abnormalities in 3-dimensional left ventricular mechanics with anthracycline chemotherapy are associated with systolic and diastolic dysfunction. JACC Cardiovasc Imaging 11:1059-1068. https://doi.org/10.1016/j.jcmg.2018.01.015

30. Li VW, Liu AP, So EK, Ho KK, Yau JP, Cheuk DK, Cheung YF (2019) Two- and three-dimensional myocardial strain imaging in the interrogation of sex differences in cardiac mechanics of longterm survivors of childhood cancers. Int J Cardiovasc Imaging 35:999-1007. https://doi.org/10.1007/s10554-019-01573-1

31. Sawaya H, Sebag IA, Plana JC, Januzzi JL, Ky B, Cohen V, Gosavi S, Carver JR, Wiegers SE, Martin RP, Picard MH, Gerszten RE, Halpern EF, Passeri J, Kuter I, Scherrer-Crosbie M (2011) Early detection and prediction of cardiotoxicity in chemotherapy-treated patients. Am J Cardiol 107:1375-1380. https://doi.org/10.1016/j. amjcard.2011.01.006

32. Stoodley PW, Richards DA, Hui R, Boyd A, Harnett PR, Meikle SR, Clarke J, Thomas L (2011) Two-dimensional myocardial strain imaging detects changes in left ventricular systolic function immediately after anthracycline chemotherapy. Eur J Echocardiogr 12:945-952. https://doi.org/10.1093/ejechocard/jer187

33. Mavinkurve-Groothuis AM, Marcus KA, Pourier M, Loonen J, Feuth T, Hoogerbrugge PM, de Korte CL, Kapusta L (2013) Myocardial 2D strain echocardiography and cardiac biomarkers in children during and shortly after anthracycline therapy for acute lymphoblastic leukaemia (all): a prospective study. Eur Heart J Cardiovasc Imaging 14:562-569. https://doi.org/10.1093/ehjci/ jes 217

34. Florescu M, Magda LS, Enescu OA, Jinga D, Vinereanu D (2014) Early detection of epirubicin-induced cardiotoxicity in patients with breast cancer. J Am Soc Echocardiogr 27:83-92. https://doi. org/10.1016/j.echo.2013.10.008

35. Tarr A, Stoebe S, Tuennemann J, Baka Z, Pfeiffer D, Varga A, Hagendorff A (2015) Early detection of cardiotoxicity by 2D and 3D deformation imaging in patients receiving chemotherapy. Echo Res Pract 2:81-88. https://doi.org/10.1530/ERP-14-0084

36. Boyd A, Stoodley P, Richards D, Hui R, Harnett P, Vo K, Marwick T, Thomas L (2017) Anthracyclines induce early changes in left ventricular systolic and diastolic function: a single centre study. PLoS One. 12:e0175544. https://doi.org/10.1371/journ al.pone. 0175544

37. Tang Q, Jiang Y, Xu Y, Xia H (2017) Speckle tracking echocardiography predicts early subclinical anthracycline cardiotoxicity in patients with breast cancer. J Clin Ultrasound 45:222-230. https ://doi.org/10.1002/jcu.22434

38. Lorenzini C, Corsi C, Aquilina M, Gardini AC, Rocca A, Frassineti L, Scarpi E, Amadori D, Lamberti C (2013) Early detection of cardiotoxicity in chemotherapy-treated patients from real-time 3D echocardiography. Comput Cardiol Zaragoza Spain IEEE. 40:249-252

39. Okuma H, Noto N, Tanikawa S, Kanezawa K, Hirai M, Shimozawa K, Yagasaki H, Shichino H, Takahashi S (2017) Impact of persistent left ventricular regional wall motion abnormalities in childhood cancer survivors after anthracycline therapy: assessment of global left ventricular myocardial performance by $3 \mathrm{D}$ speckle-tracking echocardiography. J Cardiol 70:396-401. https ://doi.org/10.1016/j.jjcc.2016.12.015

40. Miyoshi T, Tanaka H, Kaneko A, Tatsumi K, Matsumoto K, Minami H, Kawai H, Hirata K (2014) Left ventricular endocardial dysfunction in patients with preserved ejection fraction after receiving anthracycline. Echocardiography 31:848-857. https:// doi.org/10.1111/echo.12473

41. Tadic M, Genger M, Baudisch A, Kelle S, Cuspidi C, Belyavskiy E, Burkhardt F, Venneri L, Attanasio P, Pieske B (2018) Left ventricular strain in chemotherapy-naive and radiotherapy-naive patients with cancer. Can J Cardiol 34:281-287. https://doi. org/10.1016/j.cjca.2017.11.018

42. Yu HK, Yu W, Cheuk DK, Wong SJ, Chan GC, Cheung YF (2013) New three-dimensional speckle-tracking echocardiography 
identifies global impairment of left ventricular mechanics with a high sensitivity in childhood cancer survivors. J Am Soc Echocardiogr 26:846-852. https://doi.org/10.1016/j.echo.2013.04.018

43. Ho E, Brown A, Barrett P, Morgan RB, King G, Kennedy MJ, Murphy RT (2010) Subclinical anthracycline- and trastuzumabinduced cardiotoxicity in the long-term follow-up of asymptomatic breast cancer survivors: a speckle tracking echocardiographic study. Heart 96:701-707. https://doi.org/10.1136/ hrt.2009.173997

44. Lange SA, Jung J, Jaeck A, Hitschold T, Ebner B (2016) Subclinical myocardial impairment occurred in septal and anterior LV wall segments after anthracycline-embedded chemotherapy and did not worsen during adjuvant trastuzumab treatment in breast cancer patients. Cardiovasc Toxicol 16:193-206. https://doi.org/10.1007/ s12012-015-9328-9

45. Portugal G, Moura Branco L, Galrinho A, Mota Carmo M, Timoteo AT, Feliciano J, Abreu J, Duarte Oliveira S, Batarda L, Cruz Ferreira R (2017) Global and regional patterns of longitudinal strain in screening for chemotherapy-induced cardiotoxicity. Rev Port Cardiol 36:9-15. https://doi.org/10.1016/j.repc.2016.06.009

46. Perel RD, Slaughter RE, Strugnell WE (2006) Subendocardial late gadolinium enhancement in two patients with anthracycline cardiotoxicity following treatment for Ewing's sarcoma. J Cardiovasc Magn Reson 8:789-791. https://doi.org/10.1080/10976 640600737664

47. Harries I, Biglino G, Baritussio A, De Garate E, Dastidar A, Plana JC, Bucciarelli-Ducci C (2019) Long term cardiovascular magnetic resonance phenotyping of anthracycline cardiomyopathy. Int J Cardiol. https://doi.org/10.1016/j.ijcard.2019.04.026

48. Barros MVL, Macedo AVS, Sarvari SI, Faleiros MH, Felipe PT, Silva JLP, Edvardsen T (2019) Left ventricular regional wall motion abnormality is a strong predictor of cardiotoxicity in breast cancer patients undergoing chemotherapy. Arq Bras Cardiol 112:50-56. https://doi.org/10.5935/abc.20180220

49. Bogaert J, Rademakers FE (2001) Regional nonuniformity of normal adult human left ventricle. Am J Physiol Heart Circ Physiol 280:H610-620. https://doi.org/10.1152/ajpheart.2001.280.2.H610

50. Chareonthaitawee P, Kaufmann PA, Rimoldi O, Camici PG (2001) Heterogeneity of resting and hyperemic myocardial blood flow in healthy humans. Cardiovasc Res 50:151-161. https://doi. org/10.1016/s0008-6363(01)00202-4

51. Melillo G, Lima JA, Judd RM, Goldschmidt-Clermont PJ, Silverman HS (1996) Intrinsic myocyte dysfunction and tyrosine kinase pathway activation underlie the impaired wall thickening of adjacent regions during postinfarct left ventricular remodeling. Circulation 93:1447-1458. https://doi.org/10.1161/01.CIR.93.7.1447

52. Laufer-Perl M, Arnold JH, Mor L, Amrami N, Derakhshesh M, Moshkovits Y, Sadeh B, Arbel Y, Topilsky Y, Rozenbaum Z (2019) The association of reduced global longitudinal strain with cancer therapy-related cardiac dysfunction among patients receiving cancer therapy. Clin Res Cardiol. https://doi.org/10.1007/ s00392-019-01508-9

53. Fallah-Rad N, Walker JR, Wassef A, Lytwyn M, Bohonis S, Fang T, Tian G, Kirkpatrick ID, Singal PK, Krahn M, Grenier D, Jassal DS (2011) The utility of cardiac biomarkers, tissue velocity and strain imaging, and cardiac magnetic resonance imaging in predicting early left ventricular dysfunction in patients with human epidermal growth factor receptor ii-positive breast cancer treated with adjuvant trastuzumab therapy. J Am Coll Cardiol 57:22632270. https://doi.org/10.1016/j.jacc.2010.11.063

54. Sawaya H, Sebag IA, Plana JC, Januzzi JL, Ky B, Tan TC, Cohen V, Banchs J, Carver JR, Wiegers SE, Martin RP, Picard MH, Gerszten RE, Halpern EF, Passeri J, Kuter I, Scherrer-Crosbie M (2012) Assessment of echocardiography and biomarkers for the extended prediction of cardiotoxicity in patients treated with anthracyclines, taxanes, and trastuzumab. Circ Cardiovasc Imaging 5:596-603. https://doi.org/10.1161/CIRCIMAGIN G.112.973321

55. Baratta S, Damiano MA, Marchese ML, Trucco JI, Rizzo MM, Bernok F, Chejtman D, Olano D, Rojas M, Hita A (2018) Serum markers, conventional doppler echocardiography and two-dimensional systolic strain in the diagnosis of chemotherapy-induced myocardial toxicity. Rev Argent Cardiol 81:133-138. https://doi. org/10.7775/rac.v81.i2.2300

56. Muraru D, Niero A, Rodriguez-Zanella H, Cherata D, Badano L (2018) Three-dimensional speckle-tracking echocardiography: benefits and limitations of integrating myocardial mechanics with three-dimensional imaging. Cardiovasc Diag Ther 8:101-117. https://doi.org/10.21037/cdt.2017.06.01

57. Badano LP, Cucchini U, Muraru D, Al Nono O, Sarais C, Iliceto S (2013) Use of three-dimensional speckle tracking to assess left ventricular myocardial mechanics: inter-vendor consistency and reproducibility of strain measurements. Eur Heart J Cardiovasc Imaging 14:285-293. https://doi.org/10.1093/ehjci/jes 184 